\title{
Modeling of second order space charge driven coherent sum and difference instabilities
}

\author{
Yao-Shuo Yuan, ${ }^{1}$ Oliver Boine-Frankenheim, ${ }^{1,2}$ and Ingo Hofmann ${ }^{1,2}$ \\ ${ }^{1}$ Technische Universität Darmstadt, Schlossgartenstrasse 8, 64289 Darmstadt, Germany \\ ${ }^{2}$ GSI Helmholtzzentrum für Schwerionenforschung GmbH, Planckstrasse 1, 64291 Darmstadt, Germany
}

(Received 20 July 2017; published 26 October 2017)

\begin{abstract}
Second order coherent oscillation modes in intense particle beams play an important role for beam stability in linear or circular accelerators. In addition to the well-known second order even envelope modes and their instability, coupled even envelope modes and odd (skew) modes have recently been shown in [Phys. Plasmas 23, 090705 (2016)] to lead to parametric instabilities in periodic focusing lattices with sufficiently different tunes. While this work was partly using the usual envelope equations, partly also particle-in-cell (PIC) simulation, we revisit these modes here and show that the complete set of second order even and odd mode phenomena can be obtained in a unifying approach by using a single set of linearized rms moment equations based on "Chernin's equations." This has the advantage that accurate information on growth rates can be obtained and gathered in a "tune diagram." In periodic focusing we retrieve the parametric sum instabilities of coupled even and of odd modes. The stop bands obtained from these equations are compared with results from PIC simulations for waterbag beams and found to show very good agreement. The "tilting instability" obtained in constant focusing confirms the equivalence of this method with the linearized Vlasov-Poisson system evaluated in second order.
\end{abstract}

DOI: 10.1103/PhysRevAccelBeams.20.104201

\section{INTRODUCTION}

Coherent modes in intense charged particle beams are intrinsic properties of these beams, which characterize the beam's response to external perturbations or-as in our case - to parametric excitation or instability. In the latter case the modes can be excited by a small initial mismatch and amplified by the periodic focusing structure or by anisotropy, resulting eventually in emittance growth and/or beam loss. Theoretical understanding and accurate calculation of the corresponding stopbands is essential for the identification of suitable working points in accelerators for high intensity. Based on the linearized Vlasov-Poisson system of equations, coherent modes can be well-described analytically (see, for example, Refs. [1-3] as well as in simulations (for example Ref. [4,5] and a recent review [6]). Among these modes, the second order even modes, or envelope modes, are of importance since they can be observed in mismatched beams and may result in the well-known envelope instability $[2,7,8]$. The second order odd or "skew" modes [2] can drive the resonant emittance exchange by space charge coupling and trigger the "tilting instability" for sufficiently large anisotropy [9-11]. More recent studies have revealed that in periodic focusing the

Published by the American Physical Society under the terms of the Creative Commons Attribution 4.0 International license. Further distribution of this work must maintain attribution to the author(s) and the published article's title, journal citation, and DOI. second order odd mode can be excited by a parametric instability, likewise a coupled sum mode of two even envelope modes $[12,13]$. Application of these findings to a transverse-longitudinal sum envelope instability in high intensity linear accelerators has been discussed in Ref. [14].

The present study reviews the complete set of second order instabilities, including even and odd modes and their sum effects, by employing a unified second order approach. This is possible by using the self-consistent set of equations derived by Chernin [15] (written as "Chernin equations" in the following). Note that these equations are equivalent to forming second order moments of the full Vlasov-Poisson equations in $4 \mathrm{~d}$ phase space. In the absence of skew terms - either external, or self-skewing - the 10 Chernin equations reduce to the well-known $2 \mathrm{~d}$ envelope equations of even modes.

The paper is organized as follows. We first extract the second order moment equations without external skew terms from Chernin's equations. This is followed by calculating odd or skew mode coherent frequencies in the case of both symmetric and asymmetric beams in a constant focusing structure; furthermore a comparison with earlier results derived from Vlasov's equation as well as a Fourier analysis of noise spectra from a $2 \mathrm{~d}$ particle-in-cell (PIC) simulation. Finally, we explore the theoretical mechanism of sum envelope instabilities, including both the coupled even mode sum instability and the odd mode sum instability. Full stop bands are calculated and compared with PIC simulation results including particle evolution in real space. 


\section{SECOND ORDER MOMENT EQUATIONS}

In the following we assume $x$ and $y$ are the transverse degrees in horizontal and vertical direction, respectively; $s$ the longitudinal coordinate; and $k_{0, x, y}, k_{x, y}$ are the phase advances per focusing cell without and with space charge, respectively. $\phi_{1}$ and $\phi_{2}$ denote the phase shifts of slow even and fast even modes (also called, "quadrupolar" and "breathing" modes, see Ref. [3]), while $\psi_{1}, \psi_{2}\left(\psi_{1}>\psi_{2}\right)$ are the phase shifts of the two second order odd modes, respectively. We first consider a lattice including an external skew focusing force. Following Chernin, the evolution of second order moments of the beam can be written in matrix form [15]

$$
\boldsymbol{\Sigma}^{\prime}=M \boldsymbol{\Sigma}+(M \boldsymbol{\Sigma})^{T},
$$

with

$$
M=\left(\begin{array}{cccc}
0 & 1 & 0 & 0 \\
-\kappa_{x}(s) & 0 & -q_{x y}(s) & 0 \\
0 & 0 & 0 & 1 \\
-q_{x y}(s) & 0 & -\kappa_{y}(s) & 0
\end{array}\right)
$$

Here, " " denotes the derivative with respect to $s$, the superscript $T$ means the transposed matrix, $\boldsymbol{\Sigma}$ represents the $4 \times 4$ matrix of the second order moments, with elements $\Sigma_{i j}$ defined by $\Sigma_{i j} \equiv\left\langle\nu_{i} \nu_{j}\right\rangle-\left\langle\nu_{i}\right\rangle\left\langle\nu_{j}\right\rangle$, where the averages are taken over the phase space variables and the subscripts $i, j$ run from 1 to 4 representing $x, x^{\prime}, y, y^{\prime}$. $\kappa_{x}=\kappa_{x 0}-\Delta \kappa_{x}, \kappa_{y}=\kappa_{y 0}-\Delta \kappa_{y}$ and $q_{x y}=q_{x y, 0}-\Delta q_{x y}$, with $\kappa_{x 0}=(1 / B \rho)\left(\partial B_{y} / \partial x\right), \kappa_{y 0}=-(1 / B \rho)\left(\partial B_{x} / \partial y\right)$ the external focusing gradients and $q_{x y 0}=(1 / B \rho)\left(\partial B_{x} / \partial x\right)$ the external linear coupling, ( $B \rho$ is the magnetic rigidity) and $\Delta \kappa_{x}, \Delta \kappa_{y}$ and $\Delta q_{x y}$ the space charge induced shifts, defined by

$$
\begin{aligned}
\Delta \kappa_{x} & =\frac{K}{2} \frac{S_{y}}{S_{0}\left(S_{x}+S_{y}\right)}, \quad \Delta \kappa_{y}=\frac{K}{2} \frac{S_{x}}{S_{0}\left(S_{x}+S_{y}\right)}, \\
\Delta q_{x y} & =\frac{K}{2} \frac{\Sigma_{13}}{S_{0}\left(S_{x}+S_{y}\right)},
\end{aligned}
$$

using

$$
\begin{aligned}
& S_{0}=\sqrt{\Sigma_{11} \Sigma_{33}-\Sigma_{13}^{2}}, \\
& S_{x}=\Sigma_{11}+S_{0}, \quad S_{y}=\Sigma_{33}+S_{0} .
\end{aligned}
$$

Here $K$ is the space charge perveance parameter, defined by $K=2 N_{L} r_{c} /\left(\beta^{2} \gamma^{3}\right)$, with $N_{L}$ the number of particles per length, $r_{c}$ the classical proton radius, $\beta$ and $\gamma$ the relativistic factors.

Clearly, $\Sigma_{13}, \Sigma_{14}, \Sigma_{23}$ and $\Sigma_{24}$ denote the coupling moments between $x$ and $y$. Equation (1) can be also written in concise form [16]

$$
\boldsymbol{\Sigma}^{\prime}=G(\boldsymbol{\Sigma}(s), s)
$$

For a lattice with constant focusing and coupling, Eq. (5) can be readily solved with a given initial generalized emittance [17]. For an alternating focusing lattice, matched solutions $\boldsymbol{\Sigma}_{\mathbf{0}}(s)$ can be obtained numerically from Eq. (5) with periodic conditions $\boldsymbol{\Sigma}_{\mathbf{0}}(s)=\boldsymbol{\Sigma}_{\mathbf{0}}(s+L)$, where $L$ is the periodicity.

Let us assume a slightly mismatched case, where the moments can be written as $\boldsymbol{\Sigma}(s)=\boldsymbol{\Sigma}_{\mathbf{0}}(s)+\boldsymbol{\Sigma}_{\mathbf{p}}(s)$ ("p" denoting a small perturbation of the matched solution). After Taylor expanding and keeping only first order terms we have

$$
\boldsymbol{\Sigma}^{\prime}=\left(\boldsymbol{\Sigma}_{\mathbf{0}}+\boldsymbol{\Sigma}_{\mathbf{p}}\right)^{\prime}=\mathbf{G}\left(\boldsymbol{\Sigma}_{\mathbf{0}}\right)+\mathbf{J}\left(\boldsymbol{\Sigma}_{\mathbf{p}}\right),
$$

which can be divided into two parts

$$
\Sigma_{0}^{\prime}=\mathbf{G}\left(\Sigma_{0}\right), \quad \Sigma_{\mathbf{p}}^{\prime}=\mathbf{J}\left(\Sigma_{0}\right) \Sigma_{\mathbf{p}}
$$

Here $\mathbf{J}$ represents the Jacobian matrix of $\mathbf{G}$ with elements $J_{k, l}=\partial G_{k}\left(\Sigma_{l}\right) / \partial \Sigma_{l}(k, l$ run from 1 to 10 along the possible combinations of the second order moments $\Sigma_{i j}$.).

Next we consider a lattice without external coupling force $\left(q_{x y, 0}=0\right)$. Furthermore, we note that the moments $\Sigma_{22}$ and $\Sigma_{44}$ can be replaced by the rms emittance with $\varepsilon_{x}=\sqrt{\Sigma_{11} \Sigma_{22}-\Sigma_{12}^{2}}$ and $\varepsilon_{y}=\sqrt{\Sigma_{33} \Sigma_{44}-\Sigma_{34}^{2}}$ [18]. With these conditions the equations of the matched moments in Eqs. (7) can be simplified to

$$
\begin{aligned}
& \Sigma_{11}^{\prime}=2 \Sigma_{12}=G_{1}, \\
& \Sigma_{12}^{\prime}=\frac{\epsilon_{x}^{2}+\Sigma_{12}^{2}}{\Sigma_{11}}-k_{x} \Sigma_{11}=G_{2}, \\
& \Sigma_{13}^{\prime}=\Sigma_{23}+\Sigma_{14}=G_{3}, \\
& \Sigma_{14}^{\prime}=\Sigma_{24}-k_{y} \Sigma_{13}+q_{x y} \Sigma_{11}=G_{4}, \\
& \Sigma_{23}^{\prime}=\Sigma_{24}-k_{x} \Sigma_{13}+q_{x y} \Sigma_{33}=G_{5}, \\
& \Sigma_{24}^{\prime}=-k_{x} \Sigma_{14}-k_{y} \Sigma_{23}+q_{x y}\left(\Sigma_{12}+\Sigma_{34}\right)=G_{6}, \\
& \Sigma_{33}^{\prime}=2 \Sigma_{34}=G_{7}, \\
& \Sigma_{34}^{\prime}=\frac{\epsilon_{y}^{2}+\Sigma_{34}^{2}}{\Sigma_{33}}-k_{y} \Sigma_{33}=G_{8} .
\end{aligned}
$$

Here $G_{1}$ to $G_{8}$ denote the row elements of the matrix $\mathbf{G}$, respectively. For the matched case we assume that the coupling moments are zero: $\Sigma_{13,0}=\Sigma_{14,0}=\Sigma_{23,0}=$ $\Sigma_{24,0}=0$ [19] (In Ref. [19], it is alternatively assumed that non-zero value for $\Sigma_{14,0}$ and $\Sigma_{23,0}$ with $\Sigma_{14,0}=-\Sigma_{23,0}$ ), and Eqs. (7) are simplified to the envelope equations 


$$
\begin{aligned}
& \Sigma_{11,0}^{\prime}=2 \Sigma_{12,0}, \\
& \Sigma_{12,0}^{\prime}=\frac{\epsilon_{x}^{2}+\Sigma_{12,0}^{2}}{\Sigma_{11,0}}-k_{x} \Sigma_{11,0}, \\
& \Sigma_{33,0}^{\prime}=2 \Sigma_{34,0}, \\
& \Sigma_{34,0}^{\prime}=\frac{\epsilon_{y}^{2}+\Sigma_{34,0}^{2}}{\Sigma_{33,0}}-k_{y} \Sigma_{33,0},
\end{aligned}
$$

with the corresponding perturbation equations

$$
\begin{aligned}
& \Sigma_{11}^{p^{\prime}}=2 \Sigma_{12}^{p} \\
& \Sigma_{12}^{p^{\prime}}=\left(\frac{\partial G_{2}}{\partial \Sigma_{11}}\right)_{0} \Sigma_{11}^{p}+\left(\frac{\partial G_{2}}{\partial \Sigma_{12}}\right)_{0} \Sigma_{12}^{p}+\left(\frac{\partial G_{2}}{\partial \Sigma_{33}}\right)_{0} \Sigma_{33}^{p} \\
& \Sigma_{33}^{p^{\prime}}=2 \Sigma_{34}^{p} \\
& \Sigma_{34}^{p^{\prime}}=\left(\frac{\partial G_{8}}{\partial \Sigma_{11}}\right)_{0} \Sigma_{11}^{p}+\left(\frac{\partial G_{8}}{\partial \Sigma_{33}}\right)_{0} \Sigma_{33}^{p}+\left(\frac{\partial G_{8}}{\partial \Sigma_{34}}\right)_{0} \Sigma_{34}^{p} .
\end{aligned}
$$

The two second order even modes (fast and slow modes) can be obtained from the above equations. In order to calculate the odd modes, we need to derive the perturbation equations of coupling moments. Based on Eqs. (8) we obtain

$$
\begin{aligned}
\Sigma_{13}^{p \prime} & =\Sigma_{14}^{p}+\Sigma_{23}^{p}, \\
\Sigma_{14}^{p \prime} & =\left(\frac{\partial G_{4}}{\partial \Sigma_{13}}\right)_{0} \Sigma_{13}^{p}+\Sigma_{24}^{p}, \\
\Sigma_{23}^{p \prime} & =\left(\frac{\partial G_{5}}{\partial \Sigma_{13}}\right)_{0} \Sigma_{13}^{p}+\Sigma_{24}^{p}, \\
\Sigma_{24}^{p \prime} & =\left(\frac{\partial G_{6}}{\partial \Sigma_{13}}\right)_{0} \Sigma_{13}^{p}+\left(\frac{\partial G_{6}}{\partial \Sigma_{14}}\right)_{0} \Sigma_{14}^{p}+\left(\frac{\partial G_{6}}{\partial \Sigma_{23}}\right)_{0} \Sigma_{23}^{p} .
\end{aligned}
$$

Note that in the matched case, $\Sigma_{13,0}=\Sigma_{14,0}=\Sigma_{23,0}=$ $\Sigma_{24,0}=0$, and from Eqs. (3) and Eqs. (4) we have

$$
\begin{aligned}
\left(\frac{\partial \Delta q_{x y}}{\partial \Sigma_{11}}\right)_{0} & =\left(\frac{\partial \Delta q_{x y}}{\partial \Sigma_{33}}\right)_{0}=0, \\
\frac{\partial \Delta q_{x y}}{\partial \Sigma_{13}} & =\frac{K}{2} \frac{1}{S_{0}\left(S_{x}+S_{y}\right)} .
\end{aligned}
$$

The Eqs. (12) mean that the space charge coupling term has no influence on the matched solution, but on the perturbation second order odd modes. With the matched beam moments from Eqs. (9), the perturbations in Eqs. (10) and Eqs. (11) can be used to calculate the two even as well as the two odd modes.

\section{ODD MODES AND TILTING INSTABILITY IN CONSTANT FOCUSING}

As an application and a test of the above set of equations, we calculate the two odd mode phase advances for a constant focusing structure. In constant focusing Eqs. (11) take the form

$$
\begin{aligned}
& \frac{\mathrm{d}^{2}}{\mathrm{~d} s^{2}} \Sigma_{13}^{p}=a_{1} \Sigma_{13}^{p}+a_{2} \Sigma_{24}^{p}, \\
& \frac{\mathrm{d}^{2}}{\mathrm{~d} s^{2}} \Sigma_{24}^{p}=a_{3} \Sigma_{13}^{p}+a_{4} \Sigma_{24}^{p},
\end{aligned}
$$

with the coefficients

$$
\begin{aligned}
& a_{1}=\left[\frac{\partial q_{x y}}{\partial \Sigma_{13}}\left(\Sigma_{11}+\Sigma_{33}\right)-\left(k_{x}+k_{y}\right)\right]_{0}, \\
& a_{2}=2, \\
& a_{3}=\left[-\frac{\partial q_{x y}}{\partial \Sigma_{13}}\left(k_{x} \Sigma_{11}+k_{y} \Sigma_{33}\right)+2 k_{x} k_{y}\right]_{0}, \\
& a_{4}=-\left(k_{x}+k_{y}\right)_{0} .
\end{aligned}
$$

The two odd modes with the corresponding phase shifts $\psi_{1,2}$ can be readily decoupled and obtained from Eq. (13). For a round beam under a symmetric focusing with $k_{0, x}=k_{0, y}=k_{0}$, the results of odd modes $\psi_{1,2}$ calculated numerically from Eq. (13), along with the even ones $\phi_{1,2}$ from Eq. (10), are shown in Fig. 1 for different beam currents in units of the space charge tune depression $\eta_{x} \equiv k_{x} / k_{0 x}$. It can be seen that due to the axial symmetry the fast branch of the odd modes $\psi_{1}$ is always identical with the slow branch of the even mode, while the slow odd mode $\psi_{2}$ is zero (not shown in the plot). This is in agreement with the results obtained from Vlasov's equation for the round beam case in Ref. [3].

For a nonaxisymmetric focusing case, where the transverse beam shape becomes elliptical, the slow branch of the odd modes becomes nonzero. An example with $k_{0, x}=60^{\circ}$ and $k_{0, y}=66.7^{\circ}$ and $\epsilon_{x} / \epsilon_{y}=2$ is shown in Fig. 2. It is seen that the slow odd mode $\psi_{2}$ is close to $k_{y}-k_{x}$ for increasing beam current up to the point, where both become zero. In this region the slow odd mode is called a "difference mode." To the right side of this point in Fig. 2, $\left(\psi_{2}\right)^{2}$ becomes negative, hence unstable, and the imaginary part is shown. This "tilting instability" is discussed in detail

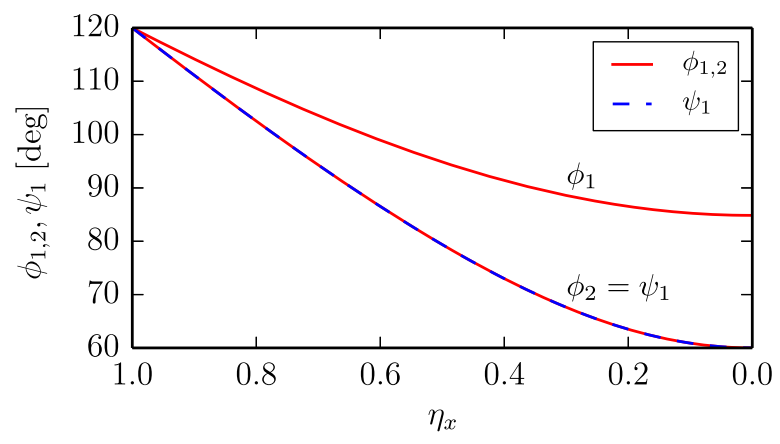

FIG. 1. Round beam case, $k_{0, x}=k_{0, y}=k_{0}=60^{\circ}$ : Phase shifts $\phi_{1,2}, \psi_{1}$ versus tune depression in $x$ in a constant focusing channel. 


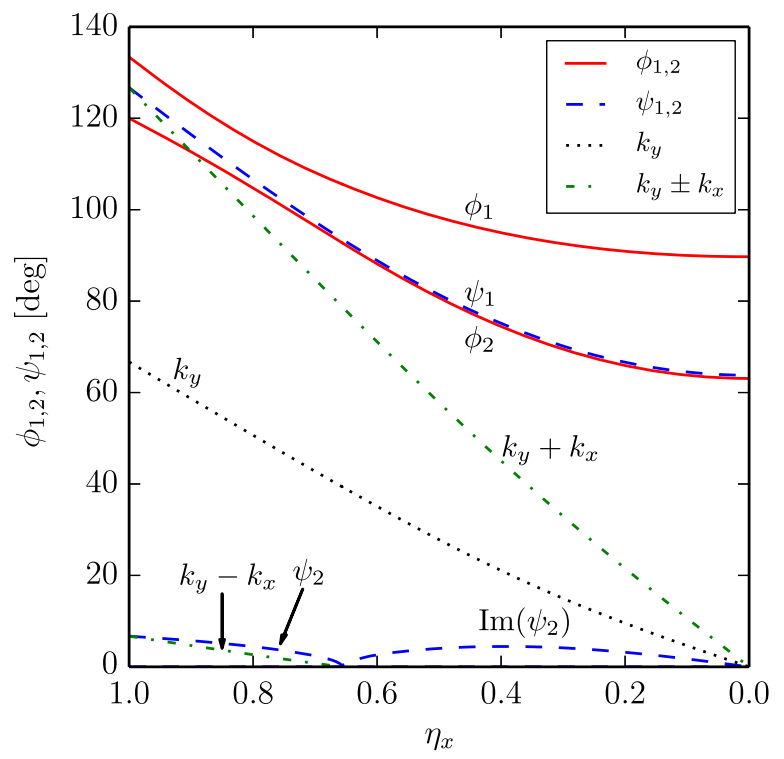

FIG. 2. Elliptical beam case, $k_{0, x}=60^{\circ}, k_{0, y}=66.7^{\circ}$ and $\epsilon_{x} / \epsilon_{y}=2$ : Phase shifts of coherent even modes $\phi_{1,2}$ and odd modes $\psi_{1,2}$ versus tune depression in $x$ in a constant focusing channel.

further below. For the fast odd mode $\psi_{1} \geq k_{x}+k_{y}$, hence this mode can be called "sum mode."

PIC simulations using PYORBIT [20] are performed to compare with results from this analytical framework. To this end 50000 particles are tracked over 1000 periodic FODO cells, and a Fourier analysis is employed on the coupling moment $\langle x y\rangle$ at various beam intensities in terms of $\eta_{x}$. As shown in Fig. 3, the beam spectra of $\langle x y\rangle$ for a KV and a waterbag distribution agree well with the results of the second order odd mode phase advances $\psi_{1,2}$ in Fig. 2 obtained from the theoretical approach of Eq. (13).

Note the minor bump to the left-hand side (l.h.s.) of the sum mode, which has a downward shift from $\psi_{1}$ increasing roughly linearly with space charge. This might be associated with single particle tunes and numerical noise of the PIC code.

An interesting property of the second order odd modes shown in Ref. [3] is found for $\epsilon_{x} \neq \epsilon_{y}$ and sufficiently large anisotropy. With an external focusing stronger in $y$ than in $x$ (or vice versa), but reverted due to space charge, the beam is subject to the tilting instability with periodical exchange of emittances as indicated above in Fig. 2. In this case the solution of Eq. (11) shows that the squared phase shift of the difference mode becomes negative, $\left(\psi_{2}\right)^{2}<0$, indicating instability. As an example, Fig. 4 illustrates the imaginary part of $\psi_{2}$ for constant $k_{y} / k_{0, y}=0.8$ and $\varepsilon_{x} / \varepsilon_{y}=2$, as a function of the betatron tune ratio, hence the stop band of the tilting instability. We find that the values of growth rates as well as the width of the stop band are in excellent agreement with the curve of the second order odd mode in Fig. 4 in Ref. [5], which is based on the

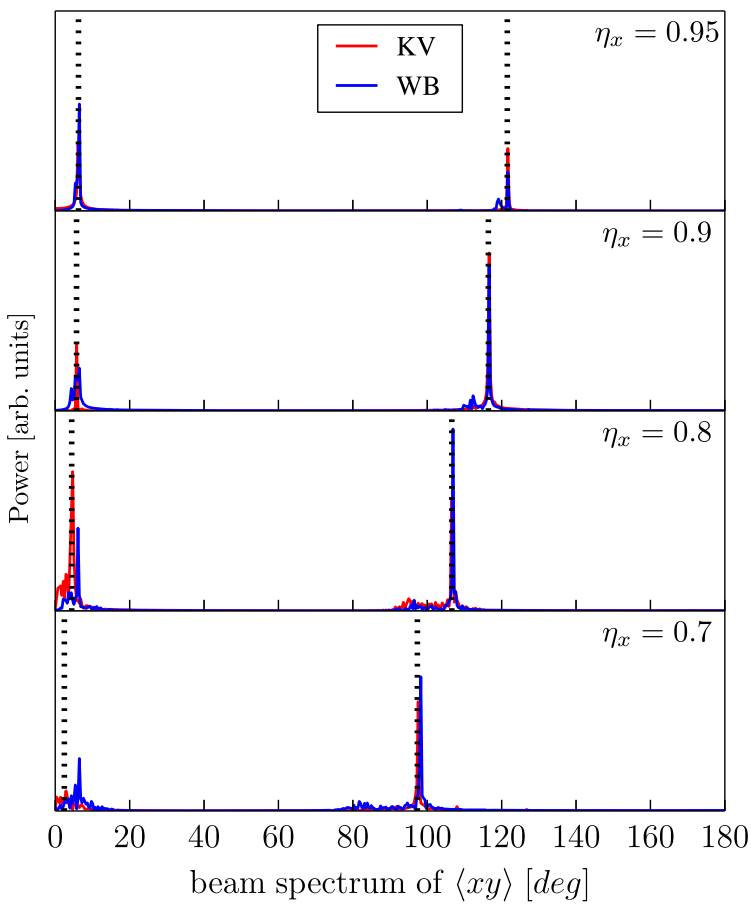

FIG. 3. Elliptical beam case, $k_{0, x}=60^{\circ}, k_{0, y}=66.7^{\circ}$ : Beam spectrum of $\langle x y\rangle$ from PIC simulation of KV distribution (red solid) and waterbag (WB) distribution (blue solid), compared with the analytical results (dotted black lines) of phase shifts of second order odd sum and difference modes $\psi_{1,2}$, for different values of $\eta_{x}$ in a constant focusing channel.

linearized Vlasov-Poisson approach - thus confirming the equivalence of these two models. Note that following Ref. [5] the tilting instability is accompanied by ausually-dominant fourth order resonance in an adjacent stop band, which is the well-known Montague instability (see also Ref. [11]).

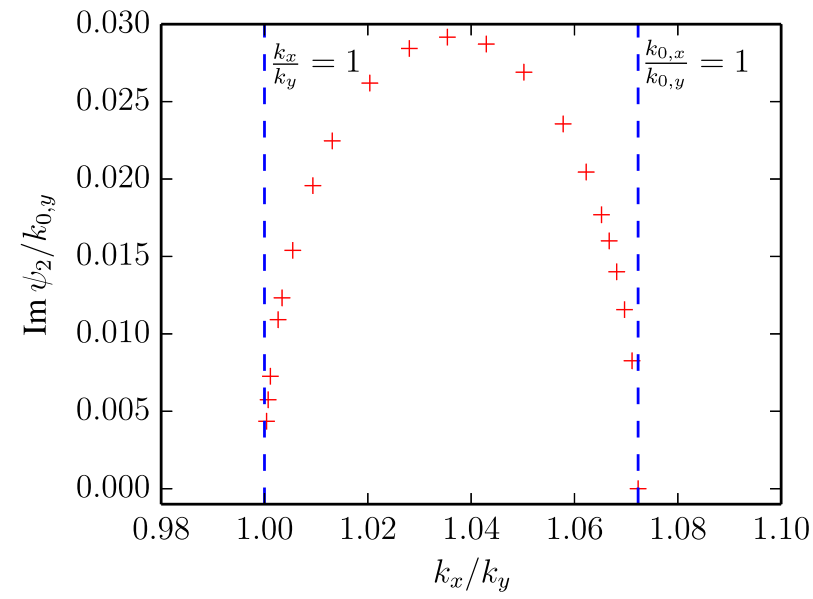

FIG. 4. Growth rate of difference odd mode (normalized) for constant $k_{y} / k_{0, y}=0.8$ and $\varepsilon_{x} / \varepsilon_{y}=2$ as a function of the betatron tune ratio, indicating the tilting instability in a constant focusing channel. 


\section{STOP BANDS OF SUM ENVELOPE INSTABILITIES IN PERIODIC FOCUSING}

The recently discovered parametric sum envelope beam instability in periodical, unsymmetrical focusing can be classified into two essentially distinct modes [12]: i. Coupled (even) mode sum instability. Based on space charge coupling between even envelope modes and their parametric resonance with the lattice, the stop band of coupled even modes is theoretically illustrated in Figs. 1, 2 of Ref. [12]. ii. Skew mode sum instability. This instability is based on parametric resonance of the odd sum mode as shown by the PIC simulation in Fig. 3 of Ref. [12]. In the following, we explore the theoretical mechanisms of both modes by using the formalism of Eqs. (11). Analogous to the treatment of the usual envelope instabilities [7,21], the four eigenvalues can be calculated by integrating Eqs. (10) and Eqs. (11): $\zeta_{m+1}=M \zeta_{m}$, where $M$ is the map for the perturbation vector $\zeta=\left(\Sigma_{11}^{p}, \Sigma_{12}^{p}, \Sigma_{33}^{p}, \Sigma_{34}^{p}, \Sigma_{13}^{p}, \Sigma_{14}^{p}, \Sigma_{23}^{p}, \Sigma_{24}^{p}\right)$ over one periodic cell. The eight eigenvalues of $M$, $\lambda_{i}=\left|\lambda_{i}\right| e^{i \phi_{i}}(i=1,2, \ldots 8)$, exist only as reciprocal or as conjugate. One of the moduli larger than unity can be used as growth factor per lattice period of the sum envelope instabilities.

The example in Fig. 5 shows the second order even and odd modes as a function of beam intensity in an

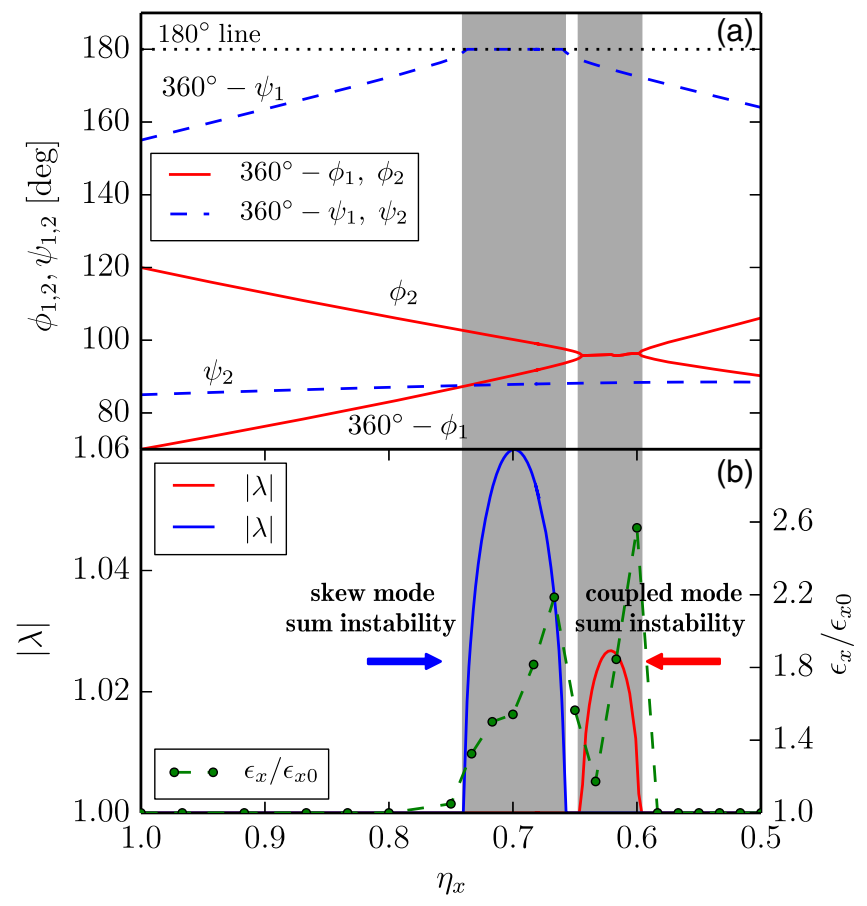

FIG. 5. Perturbation theory results for both types of sum envelope instabilities in a periodic focusing system. Upper plot (a): Phase shifts of $\phi_{1,2}, \psi_{1,2}$ verses tune depression in $x$; Lower plot (b): Corresponding growth factors per lattice period (continuous lines), and comparison with final emittance growth factors (dashed lines, normalized to initial values) for PIC simulation of same cases. unsymmetrical FODO lattice with $k_{0, x}=60^{\circ}$ and $k_{0, y}=145^{\circ}$. For convenience of plotting, in Fig. 5(a) the curves of $360^{\circ}-\psi_{1}$ and $360^{\circ}-\phi_{1}$ are plotted instead of $\psi_{1}$ and $\phi_{1}$. In the region $k_{x}=44.2^{\circ}$ to $39.6^{\circ}$ (corresponding to $\eta_{x}=0.737$ to 0.66 ) one branch of the odd mode is locked to $180^{\circ}$, with the corresponding eigenvalue larger than unity, indicating the skew mode sum instability. It is characterized by the parametric resonance between the focusing structure and the odd sum mode branch $\psi_{1}$. Further increasing the beam current, $\phi_{2}$ and $360^{\circ}-\phi_{1}$ (the curves in red color) become confluent, i.e., $\phi_{1}+\phi_{2}=360^{\circ}$, which means the two even modes are coupled and the coupled mode sum instability is excited. Note that the width of the stop band of the skew mode sum instability is wider than the one of the coupled mode sum instability, and both stop bands are only separated by a thin gap.

The above second order theory results provide no information on rms emittance growth. This requires PIC simulation with results shown by the dashed (green) lines in Fig. 5(b). The simulation results show $\epsilon_{x} / \epsilon_{x 0}$ after 500 focusing periods, at which time the emittance growth caused by the instability is already saturated. The initial particle distribution is chosen as rms-matched transverse waterbag. Two "sawtooth" like emittance growth phenomena are observed, with a width that agrees well with the stop bands obtained from perturbation theory stop bands. The shape of a "sawtooth" is caused by the self-detuning process, which is a remarkable feature of the second order coherent instabilities [6,21]. Note that the saturated PIC emittance growth factor for the coupled mode sum instability is larger than that of the skew mode sum instability, whereas the mode amplitude growth factor (per period) of the latter exceeds that of the former. Figures 6 and 7 show the PIC simulation results in real space projections at three consecutive periods at early stages of the two modes of instability. In the case of the coupled even mode sum instability the beam shows a coupled modulation of envelopes in $x$ and $y$. In contrast, the beam performs skew
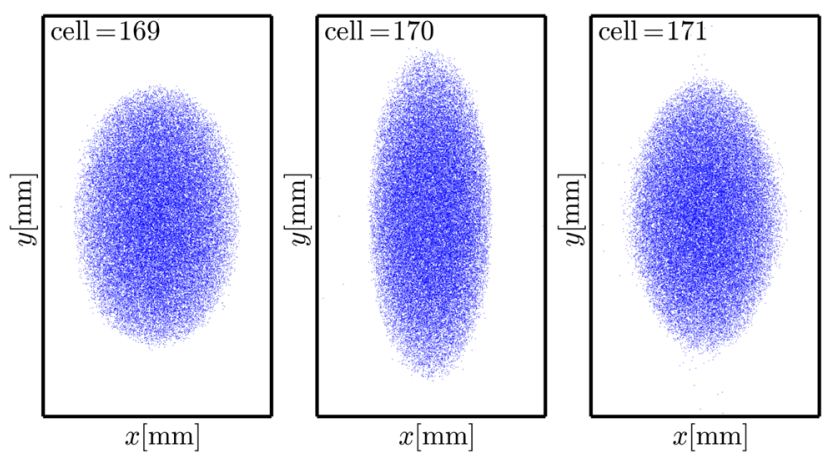

FIG. 6. Real space distributions for three consecutive cells at early stage of coupled even mode sum instability with initial $\eta_{x}=0.6$. 


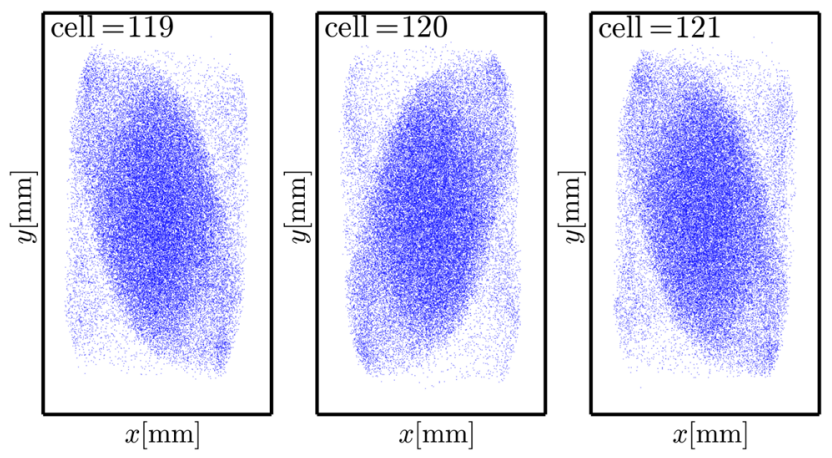

FIG. 7. Real space distribution for three representative consecutive cells at early stage of skew mode sum instability with initial $\eta_{x}=0.67$.

"swinging" in real space as a characteristic of the skew mode sum instability.

Figure 8 shows the change of phase shifts of the second order even and odd modes (in solid and dashed lines, respectively), with fixed $k_{0, y}=145^{\circ}$ and varying $k_{0, x}=$ $40^{\circ}, 50^{\circ}, 60^{\circ}$ and $70^{\circ}$. The curves of $360^{\circ}-\psi_{1}$ and $360^{\circ}-$ $\phi_{1}$ are plotted instead of $\psi_{1}$ and $\phi_{1}$. Two main properties can be observed and discussed as follows: First, with $k_{0, x}$ increasing from $40^{\circ}$ to $70^{\circ}$, the sum instability starts to appear (in the case of $k_{0, x}=50^{\circ}$ ) and then becomes enhanced (in the case of $k_{0, x}=60^{\circ}$ ); finally it becomes very weak $\left(k_{0, x}=70^{\circ}\right)$. Second, with $k_{0, x}$ getting larger, the stop band moves toward the direction of stronger space charge.

In order to present an overview on the appearance of the two kinds of sum envelope instabilities, with the

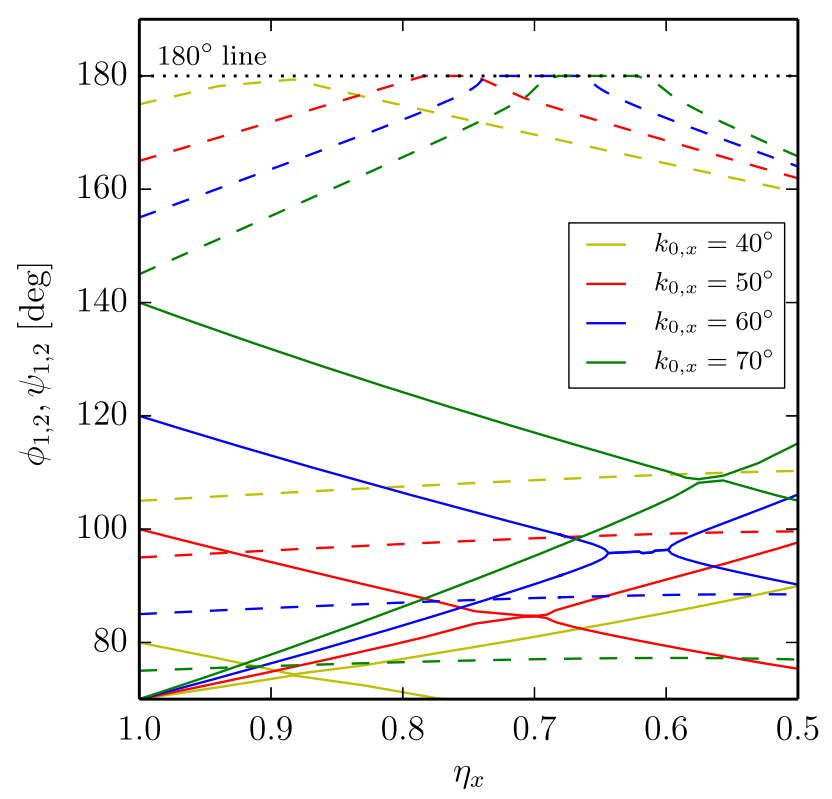

FIG. 8. Phase shifts of $\phi_{1,2}, \psi_{1,2}$ verses tune depression in $x$ with fixed $k_{0, y}=145^{\circ}$ and varying $k_{0, x}=40^{\circ}$ (yellow), $50^{\circ}$ (red), $60^{\circ}$ (blue) and $70^{\circ}$ (green).

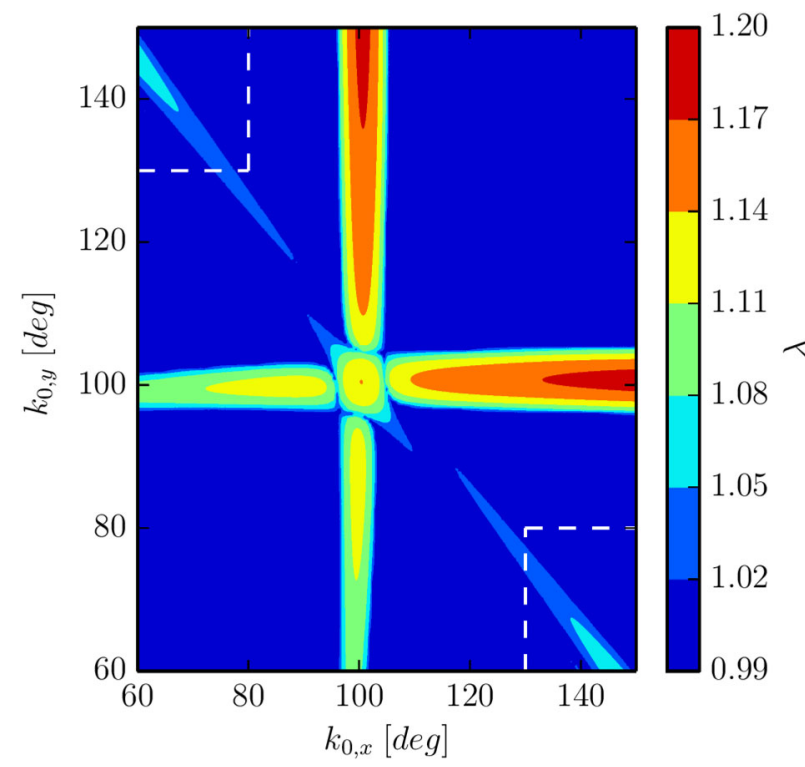

FIG. 9. Tune diagram as scan of growth factor $\lambda$ obtained from perturbation matrix.

corresponding two stop bands, a tune diagram of perturbation theory eigenvalues $\lambda$ is worked out by scanning $k_{0, x}$ and $k_{0, y}$ from $60^{\circ}$ to $150^{\circ}$ based on integrating Eqs. (10) and Eqs. (11), as shown in Fig. 9. Enlargements of the two diagonal blocks in Fig. 9 within the region of $k_{0, x}, k_{0, y}$ from $130^{\circ}$ to $150^{\circ}$ and $60^{\circ}$ to $80^{\circ}$ (marked by white dashed lines in Fig. 9) are shown in Fig. 10. We retrieve both stop bands, where the narrow one is representing the coupled even mode sum instability, and the broader one the skew mode sum instability as follows from detailed calculations of eigenvalues. Comparing with Fig. 2 in Ref. [12], the positions of stop bands for the even mode envelope instabilities and the coupled even mode sum instability are similar: the horizontal and vertical stop bands mark the $90^{\circ}$ even mode envelope instabilities, and the diagonal with negative slope denotes the stop band of the coupled even mode sum envelope instability. Note that the scan in Ref. [12] is based on envelope mismatch values, which explains the absence of the skew mode sum instability.
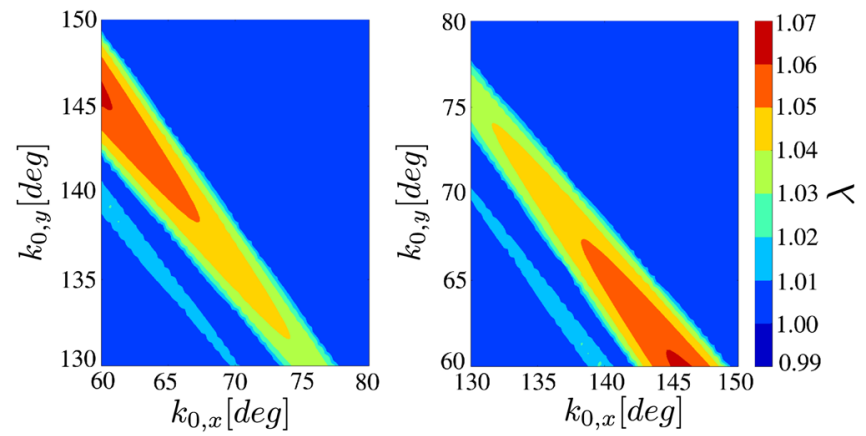

FIG. 10. Enlarged scans of the two opposite corner zones in Fig. 9. 


\section{CONCLUSIONS}

In summary, we have shown that the linearized second order moment equations derived from Chernin's equations offer a unified framework to study the stability properties of all possible second order modes, even and odd ones, in constant or periodic focusing quadrupole lattices without bending. Results are compared with earlier results on mode frequencies obtained from the linearized Vlasov-Poisson equation. Excellent agreement is found in the case of the tilting instability of odd (skew) modes in anisotropic beams and constant focusing, which confirms the equivalence of both models - on the level of second order perturbations.

Comparing moment spectra from the perturbation equations with PIC spectra for KV and waterbag beams, peaks pertaining to odd modes are retrieved at similar locations. Hence this suggests that these modes also exist for more realistic beam distributions, like waterbag.

In periodic focusing structures the stop bands of the parametric coupled even mode sum instability and the skew mode sum instability are obtained and found in very good agreement with PIC simulations, which completes the picture of second order coherent modes in $2 \mathrm{~d}$ high intensity beams. Future studies might have to include the effects of external skew magnets as well as of synchrotron motion. Correlations with the recently discovered instabilities induced by the dispersion mode in bent lattices in Ref. [21] also warrant further work.

[1] R. L. Gluckstern, Proceedings of the 1970 Proton Linear Accelerator Conference, Batavia, 1970, C700928, 811 (National Accelerator Laboratory, Batavia, 1970).

[2] I. Hofmann, L. J. Laslett, L. Smith, and I. Haber, Stability of the Kapchinskij-Vladimirskij (KV) distribution in long periodic transport systems, Part. Accel. 13, 145 (1983).

[3] I. Hofmann, Stability of anisotropic beams with space charge, Phys. Rev. E 57, 4713 (1998).

[4] A. V. Fedotov, J. A. Holmes, and R. L. Gluckstern, Instabilities of high-order beam modes driven by space-charge coupling resonances, Phys. Rev. ST Accel. Beams 4, 084202 (2001).

[5] I. Hofmann, G. Franchetti, O. Boine-Frankenheim, J. Qiang, and R. D. Ryne, Space charge resonances in two and three dimensional anisotropic beams, Phys. Rev. ST Accel. Beams 6, 024202 (2003).
[6] I. Hofmann and O. Boine-Frankenheim, Space-Charge Structural Instabilities and Resonances in High-Intensity Beams, Phys. Rev. Lett. 115, 204802 (2015).

[7] J. Struckmeier and M. Reiser, Theoretical studies of envelope oscillations and instabilities of mismatched intense charged particle beams in periodic focusing channels, Part. Accel. 14, 227 (1984).

[8] M. Tiefenback, Ph.D. thesis, Lawrence Berkeley National Laboratory Report, 1986, Report No. LBL-22465.

[9] I. Hofmann and O. Boine-Frankenheim, Resonant Emittance Transfer Driven by Space Charge, Phys. Rev. Lett. 87, 034802 (2001).

[10] G. Franchetti, I. Hofmann, and M. Aslaninejad, Collective Emittance Exchange with Linear Space Charge Forces and Linear Coupling, Phys. Rev. Lett. 94, 194801 (2005).

[11] I. Hofmann and G. Franchetti, Self-consistent study of space-charge-driven coupling resonances, Phys. Rev. ST Accel. Beams 9, 054202 (2006).

[12] O. Boine-Frankenheim, I. Hofmann, and J. Struckmeier, Parametric sum envelope instability of periodically focused intense beams, Phys. Plasmas 23, 090705 (2016).

[13] I. Hofmann and O. Boine-Frankenheim, Parametric instabilities in 3D periodically focused beams with space charge, Phys. Rev. Accel. Beams 20, 014202 (2017).

[14] I. Hofmann and O. Boine-Frankenheim, Revisiting the Longitudinal $90^{\circ}$ Limit in High Intensity Linear Accelerators, Phys. Rev. Lett. 118, 114803 (2017).

[15] D. Chernin, Evolution of rms beam envelopes in transport systems with linear xy coupling, Part. Accel. 24, 29 (1988).

[16] A. Goswami, P. Sing Babu, and V.S. Pandit, Beam dynamics and stability analysis of an intense beam in a continuously twisted quadrupole focusing channel, Eur. Phys. J. Plus (2016) 131: 393

[17] H. Qin, M. Chung, and R. C. Davidson, Generalized Kapchinskij-Vladimirskij Distribution and Envelope Equation for High-Intensity Beams in a Coupled Transverse Focusing Lattice, Phys. Rev. Lett. 103, 224802 (2009).

[18] F. J. Sacherer, RMS Envelope Equations with Space Charge, IEEE Trans. Nucl. Sci. 18, 1105 (1971).

[19] J. J. Barnard and B. Losic, in Proceedings of the 20th International Linac Conference, Monterey, CA, 2000, p. 293, http://www.slac.stanford.edu/econf/C000821/ MOE12.shtml.

[20] A. Shishlo, S. Cousineau, J. Holmes, and T. Gorlov, The Particle Accelerator Simulation Code PyORBIT, Procedia Comput. Sci. 51 (2015) 1272.

[21] Y. S. Yuan, O. Boine-Frankenheim, G. Franchetti, and I. Hofmann, Dispersion-Induced Beam Instability in Circular Accelerators, Phys. Rev. Lett. 118, 154801 (2017). 